\title{
Processus d'innovation dans les transitions agro-écologiques des pays en développement
}

\section{Innovation processes in the agro-ecological transitions in the developing}

\section{countries}

\author{
Ludovic Temple ${ }^{1}$ \\ ${ }^{1}$ Cirad, Umr Innovation, Montpellier, ludovic.temple@cirad.fr
}

\begin{abstract}
RÉSUMÉ. La problématique de ce numéro interroge les interactions entre les changements de modèles d'innovation, l'écologisation de la fonction de production et leurs conséquences sociales. Six processus d'innovation sont analysés respectivement dans les agricultures du Burkina Faso, Cameroun, Haïti, Madagascar et Sénégal. Ces situations convergent en démontrant l'utilité pour le développement de modèles d'innovation collaboratifs qui adaptent les processus étudiés aux besoins locaux. Ces modèles impliquent plus d'engagement des politiques publiques d'innovation. ABSTRACT. This article discusses the interactions between changes in models of innovation, the greening of production and the social consequences of these. Six innovation processes are analyzed in the agriculture of Burkina Faso, Cameroon, Haiti, Madagascar and Senegal, respectively. These situations coincide in demonstrating that collaborative innovation models are particularly useful for development, as they adapt the process studied to local needs. These models involve more commitment from public policy innovation.
\end{abstract}

MOTS-CLÉS. Modèle innovation, Secteur agricole et alimentaire, Agro-écologie, Biotechnologie, Pays en développement.

KEYWORDS. Model innovation, Agriculture and food sector, Agroecology, Biotechnology, country developing.

L'accroissement des inégalités mondiales de développement, les interrogations ouvertes par l'accélération du changement climatique, les nouvelles crises alimentaires et les transitions techniques : numérique, énergétique, biotechnologique convergent pour un changement de paradigme technologique dans l'agriculture l'alimentation. Cette acception reconnue dans les enceintes politiques internationales (Banque mondiale, Unesco, OCDE..) ouvre une controverse sur le modèle économique qu'elle mobilise ou renforce.

Le premier modèle repose sur l'industrialisation de la fonction de production par la standardisation des intrants utilisés pour produire des biens agricoles et alimentaires. Il est dominant dans les pays de l'OCDE. Il est pour partie issue des progrès techniques de l'après-guerre : chimie, motorisation, progrès génétique... Il repose sur la recherche d'économies d'échelle, la concentration et la spécialisation des exploitations et des territoires. Il est souvent associé à une vision moderniste car fondé sur les nouvelles techniques qui met en œuvre le progrès scientifique via les firmes mondiales de l'agrochimie et de l'agro-alimentaire. En soit il prolonge dans l'agriculture le mythe «développementaliste» du rattrapage des pays du sud grâce à l'industrie et aux transferts technologiques exogènes (COURLET et JUDET 1986). A l'extrême, il « artificialise » l'agriculture en la « désincarnant » de sa relation à la terre, au climat, au travail (humain et animal) : hydroponie, ou décébration des animaux. Ce modèle fortement efficace en termes de productivité ou de rentabilité des capitaux investis, sous-tend une trajectoire d'innovation polarisée par l'intensification de la fonction de production (plus d'intrants et de capital en substitution au travail et à la terre). L'affranchissement de l'activité de production de l'écosystème naturel et social réduit la diversité de ces derniers à une contrainte qu'il faut homogénéiser (VANLOQUEREN et BARRET 2009). Les gigantesques incendies des tourbières de Sumatra (Asie) asséchées pour produire de l'acacia destiné à l'industrie du papier ou le processus de déshumanisation du Chaco (Amérique du Sud) pour produire du soja en sont parfois des conséquences. 
Un deuxième modèle économique (SOURISSEAU et al., 2014) sous-tend une agriculture basée sur des modes de productions familiales. Il reste encore dominant dans les agricultures des pays en développement. Il se fonde d'avantage sur l'exploitation des potentialités d'innovation des écosystèmes naturels, sociaux. Il s'appuie sur les structures sociales de production prise dans leur diversité au regard de leurs ancrages historiques. Il interpelle et mobilise les capacités de la science et la technologie à l'accompagnement de cette diversité qui devient une ressource pour l'innovation. La trajectoire d'intensification qualifiée parfois d'écologique (GRIFFON, 2002) mobilise en priorité l'optimisation des potentialités des écosystèmes en hybridant les connaissances issues du progrès scientifique et les systèmes de connaissance des sociétés rurales et entrepreneuriales localisées. A l'extrême, elle renvoie à la permaculture ou d'autres formes d'agricultures biologiques fondées sur l'autoproduction d'intrants.

Ces deux modèles « stylisés » cohabitent dans les différentes réalités agraires du nord et du sud. Ils sont en concurrence dans la captation des ressources (terre, eau, travail, connaissance, financement) ou dans l'obtention du soutien des politiques publiques d'innovation et de recherche. Dans certaines situations ces modèles sont en complémentarité. Ils convergent ainsi dans la reconnaissance du caractère inadapté du modèle d'innovation linéaire diffusionniste de conception de l'invention par la recherche scientifique et de son transfert du laboratoire aux agricultures mondiales (MEYNARD et al., 2016). Cette convergence se révèle par la montée en puissance dans les politiques d'innovation et de recherche du référentiel d'analyse systémique de l'innovation qui peut alternativement être mobilisé pour améliorer les conditions d'usage des biotechnologies ou accompagner l'innovation issue des savoirs et connaissances tacites des sociétés rurales (TOUZARD et al., 2015).

Ce numéro spécial met en débat dans différentes situations les articulations entre le changement de modèle d'innovation et les trajectoires technologiques qui intensifient durablement la production agricole. Ces trajectoires sont principalement fondées sur des mécanismes d'écologisation de la fonction de production et de diminution dans l'usage d'intrants de synthèse. Les conséquences socioéconomiques sur les agricultures des pays en développement y sont partiellement abordées.

Eveline Compaoré et al. au Burkina Faso, partant des promesses technologiques de diminution de pesticides par la diffusion des OGM d'une firme agro-chimique, montrent comment les lobbies liés aux investissements globalisés sur les OGM créent les conditions institutionnelles de vulgarisation du coton Bt. Elle analyse comment l'affranchissement des principes de précaution et de prise en compte des attentes des parties prenantes contribuent à l'échec du processus d'innovation. Elle en interroge les conséquences sociales négatives que réalise une telle trajectoire.

Salif Derra, sur les conditions de développement de la culture du jatropha pour produire de l'énergie toujours au Burkina Faso, prolonge l'interrogation. Il montre comment la structuration des investissements de recherche et entrepreneuriaux dans le secteur des bioénergies institue des dynamiques technologiques au regard de besoins définis par les pays industriels. Il souligne cependant comment elle peut aussi nourrir une diversité de modèles technologiques possibles dont certains peuvent répondre (sous certaines conditions) à des besoins localisés.

Eric Penot et al. à Madagascar questionnent comment un modèle diffusionniste d'un nouveau système de culture agro-écologique (SCV) par un projet de développement renforce son efficacité en intégrant la participation des agriculteurs dans les mécanismes d'évaluation et d'expérimentation. Les résultats soulignent pourtant des taux d'adoption faibles ou des mécanismes d'adoption partiels de ces techniques. Ils questionnent la durabilité future des adoptions au regard de la capacité à intégrer la complexité des variables institutionnelles et organisationnelles qui au-delà des échelles individuelles, structurent des coordinations de l'action collective communautaires ou professionnelles.

Euphrasie Angbo et al. en Côte d'Ivoire montrent comment un transfert technologique « sud-sud » fondé sur de nouveaux hybrides de banane plantain résistantes à des maladies (qui diminuent 
potentiellement l'usage de pesticides) mobilise des dispositifs participatifs d'évaluation multi-acteurs. Ces dispositifs solidifient un modèle d'innovation collaboratif. Ils génèrent des adaptations collectives des relations entre la recherche et les agriculteurs. Ces plateformes expérimentales adaptent l'offre de transfert technologique aux besoins des différents acteurs des chaînes de valeurs localisées.

P. Dugué et al. au Sénégal sur le maraîchage montrent comment la proximité spatiale entre différents modes de production agro-industriels et familiaux peut entrainer des processus d'innovations pour les petits producteurs. Elle favorise dans la situation observée la mutualisation de capacités expérimentales et d'apprentissage qui permet l'adoption de nouveautés issues de l'industrie (pompes thermiques ou électriques) pour l'irrigation goutte à goutte. Ce modèle d'adoption est couplé avec d'autres processus d'innovation plus fondés sur des ressources locales naturelles et cognitives, par exemple pour la fertilisation des cultures ou la valorisation optimale de la terre (associations de cultures). L'hybridation des bases de connaissances entre les organisations professionnelles, la société civile (ONG porteuse du modèle d'agriculture biologique), les entreprises et la recherche sont à la base de ces mécanismes d'innovation hybridant différents artefacts techniques et connaissances locales.

James Boyer et al. en Haïti analyse sur la production d'ignames dans le long terme comment un modèle d'innovation diffusionniste linéaire c'est transformé en un modèle ouvert et collaboratif . Il souligne en quoi cette transformation à créer et été renforcée par les synergies positives entre les mécanismes d'adoption, l'écologisation autonome du processus d'innovation, et les impacts socioéconomiques au niveau macro-économique.

Ces six articles mettent en connaissance de manière transversale des résultats qui illustrent la coévolution entre l'adaptation des modèles d'innovation en agriculture et les des trajectoires technologiques qui écologisent les pratiques agronomiques dans le contexte des agricultures intertropicales de pays en développement. Toutes les études de cas confirment l'intégration croissante de recherches participatives pour créer des interactions entre les acteurs. Ces intégrations sont plus ou moins inclusives d'acteurs locaux selon les phases du processus: conception, expérimentation, dissémination. Dans certains cas l'ouverture des modèles diffusionnistes est un simple ajustement qui modifie peu la dimension linéaire du transfert technologique gouvernée par la firme agro-chimique, ou le chercheur exogène aux sociétés rurales. Il en résulte des échecs couteux pour la population locale ou des adoptions partielles peu stabilisées. Dans d'autres cas, les outils mis en œuvre (plateformes d'innovation) modifient le schéma linéaire initial de transfert technologique. Enfin dans d'autres situations, la mise en œuvre explicite de modèles d'innovations collaboratifs dans toutes les phases du processus favorise des trajectoires de réappropriation technologique par les agriculteurs notamment dans l'autoproduction des intrants utilisés. Ces dernières situations de ruptures du modèle linéaire mettent en exergue des adoptions et des impacts de l'innovation plus affirmés au regard des bases de données mobilisés. Du point de vue de l'écologisation de la fonction de production, certaines études de cas montrent que les processus d'innovation fondés sur la réduction de la pénibilité du travail (irrigation, multiplication de semences in vivo) réalisent mieux l'implication des agriculteurs dans les mécanismes d'adoption et de dissémination des technologies. En revanche, les innovations basées sur des transferts à partir de nouvelles variétés ou des systèmes de culture mis au point dans les agricultures intensives pour réduire l'usage de pesticides sont plus controversées ou en échecs publics. De manière générale, l'ensemble de situations étudiées concerne des agricultures familiales. L'exemple du Sénégal révèle une complémentarité explicite entre différents modèles économiques d'organisation de la production. Ces études de cas se différentient dans le degré d'implication des parties prenantes et en fonction du rôle plus ou moins affirmé des incitations institutionnelles portées par les pouvoirs publics. Elles interpellent l'implication des parties prenantes dans l'évaluation d'impact des technologies (TEMPLE et al., 2016). L'élaboration de politiques d'innovation et de recherche focalisées par le bien public au regard des attentes sociétales locales est une nécessité dans le contexte institutionnel parfois fragile des pays en développement. Bien que ces résultats soient contextuels au panel d'étude de cas, le repérage de leurs convergences démontre l'utilité pour le développement de l'ouverture collaborative des modèles d'innovation mobilisés par la recherche agronomique. 


\section{Références}

COURLET C., JUDET P., «Indutrialisation et développement : la crise des paradigmes». Tiers Monde, vol. 27, no. 107, p, 519-536, 1986.

GRIFFON M., « Révolution Verte, Révolution Doublement Verte Quelles technologies, institutions et recherche pour les agricultures de l'avenir? », Mondes en développement, n¹17, p. 39-44. 1982. DOI 10.3917/MED.117.0039.

MEYNARD JM., JEUFFROY MH., LE BAIL M., LEFEVRE A., MAGRINI MB., MICHON C. ; «Designing coupled innovations for the sustainability transition of agrifood systems» Agricultural Systems, 2016. http://dx.doi.org/10.1016/j.agsy.2016.08.002.

TOUZARD JM., TEMPLE L., FAURE G., TRIOMPHE B. «Innovation systems and knowledge communities in the agriculture and agrifood sector: a literature review». Journal of Innovation Economics and Management, ${ }^{\circ} 2,17, \mathrm{p} .:$ 117-142, 2015.

SOURISSEAU J. M.. Agricultures familiales et mondes à venir. Editions QUAE 2014

VANLOQUEREN G., BARET P.V., «How agricultural research systems shape a technological regime that develops genetic engineering but locks out agroecological innovations» Research Policy, $n^{\circ}$ 38, 6 , p 971-983, 2009.

TEMPLE L., BIÉNABE E., BARRET D., SAINT-MARTIN G., « Methods for assessing the impact of research on innovation and development in the agriculture and food sectors». African Journal of Science, Technology, Innovation and Development, $\mathrm{n}^{\circ}$ 8, 5-6, p 399-410, 2016. http://dx.doi.org/10.1080/20421338.2016.1219484 